\title{
WEIGHTED LAWS OF LARGE NUMBERS FOR A CLASS OF INDEPENDENT SUMMANDS
}

\author{
BY
}

\author{
ANTHONY G. PA KES (PERTh)
}

\begin{abstract}
This paper obtains a necessary and sufficient condition for a weak law of large numbers for weighted averages of positive-valued independent random variables whose distributions belong to a class which includes the $F^{\alpha}$-scheme of record theory. Additional general conditions are found under which the weak law extends to a strong law with the same norming. Examples show these conditions can be fulfilled, and that if they are not, then the weighted averages exhibit multiple growth rates.
\end{abstract}

2020 Mathematics Subject Classification: Primary 60F05, 60F15; Secondary 60E10.

Key words and phrases: weak and strong laws of large numbers, weighted sums, relative stability, regular variation.

\section{INTRODUCTION}

Generalising Theorem 3.1 of Nakata [7], Adler and Pakes [2] obtain a weak law of large numbers (WLLN) for averages $\bar{W}_{n}=b_{n}^{-1} \sum_{j=1}^{n} Y_{j}$, where the norming constants $b_{n}$ diverge to infinity and the $Y_{j}$ are positive-valued, independent and with possibly different distributions, as follows. Denote the survivor function of $Y_{j}$ by $\bar{F}_{j}(x)=1-F_{j}(x)$ and assume there are positive constants $a_{j}$ and a survivor function $\bar{F}(x)$ such that the following integrated tail equivalence condition holds: There is a positive integer $j^{\prime}$ such that

$$
\int_{0}^{x} \bar{F}_{j}(y) d y \sim a_{j} \int_{0}^{x} \bar{F}(y) d y \quad(x \rightarrow \infty),
$$

uniformly with respect to $j \geqslant j^{\prime}$. We explain this more fully in the next section. The specification used in Nakata [7] is equivalent to assuming that $a_{j} \in(0,1]$ and setting

$$
\bar{F}_{j}(x)=a_{j} /(1+x) \quad \text { if } x>0
$$


i.e., a Pareto distribution possibly with a point mass component at the origin.

Adler and Pakes [2] show under the additional assumptions that if the distribution function $F$ is relatively stable (Bingham et al. [4, p. 372]), written

$$
F \in R S
$$

and if the constants in 1.1 satisfy

$$
\sum_{j=1}^{\infty} a_{j}=\infty
$$

then there exist norming constants $b_{n}$ such that $\bar{W}_{n} \stackrel{p}{\rightarrow} 1$. The second condition is necessary: If $\sum_{j} a_{j}<\infty$, then the random series $\sum_{j} Y_{j}$ is almost surely convergent.

Nakata [8] extends his earlier result in another direction by considering averages of weighted sums, $\bar{W}_{n}(\omega):=b_{n}^{-1} \sum_{j=1}^{n} \omega_{j} Y_{j}$ where $\left(\omega_{j}\right)$ is a set of positive weights. He shows that if $(1.2)$ holds then the conditions

$$
\lim _{n \rightarrow \infty} b_{n}^{-1} \sum_{j=1}^{n} a_{j} \omega_{j}=0
$$

and

$$
\lim _{n \rightarrow \infty} b_{n}^{-1} \sum_{j=1}^{n} a_{j} \omega_{j} \log \left(1+b_{n} / \omega_{j}\right)=A \in(0, \infty)
$$

imply that

$$
\bar{W}_{n}(\omega) \stackrel{p}{\rightarrow} A
$$

Our objective in this paper is to merge the generalisation of Adler and Pakes [2] with that of Nakata [8] to give a weak law (Theorem 2.1) under the conditions (1.1) and (1.3). This will result in a weakening of the requirement (1.4) and an extension of (1.5) to a necessary and sufficient condition, (2.5) below. In the case of constant weights, this condition simplifies to outcome (2.6) in Adler and Pakes [2] which, given their assumptions, is necessary and sufficient for their weak law.

Our condition (2.5) appears resistant to simplification without more structure. In Corollary 2.1 we give conditions under which $(2.5)$ is equivalent to the simpler condition 2.9), implying the 'explicit' form 2.10 for the norming sequence directly constructed from $\ell$ and the sums $\mathcal{A}_{n}=\sum_{j=1}^{n} a_{j} \omega_{j}$. This achieves what amounts to a formal generalisation of the weak law in Adler and Pakes [2]. The simplicity of 2.10) invites a search for more general conditions under which 2.5) and (2.10) are consistent.

Theorem 2.2 asserts the weak law with the norming 2.10$)$ under an additional condition, 2.12) below, which limits the rate at which the weights $\omega_{n}$ tend to 0 (or 
to $\infty$ ) in relation to the rate at which $\mathcal{A}_{n} \rightarrow \infty$. Theorem 2.3 gives two technical conditions (2.23) and (2.24) implying (2.5) and (2.10).

What can be said if the limit (2.12) is positive or infinite? This is explored in the context of Examples 2.1 and 2.2 in which $\omega_{n} \rightarrow 0$ and there are three cases, numbered 1 to 3 , according as the limit (2.12) is zero (as stated there), infinite, or positive and finite. The weak law holds in all cases, but for Cases 2 and 3, the form of the norming constants differs from 2.10).

Is there a strong law corresponding to Theorem 2.1? Nakata [8] answers 'yes' for his Pareto summands (his Theorem 1.4). In our notation, he assumes that the sequences $\left(a_{n}\right),\left(\omega_{n}\right)$ and $\left(b_{n}\right)$ satisfy the condition

$$
\sum_{n=1}^{\infty} \frac{a_{n} \omega_{n}}{b_{n}}<\infty
$$

(his (15)). Then the strong law is valid if a modified form of 1.57 holds, i.e., if

$$
\lim _{n \rightarrow \infty} b_{n}^{-1} \sum_{j=1}^{n} a_{j} \omega_{j} \log \left(1+b_{j} / \omega_{j}\right)=A \in(0, \infty),
$$

then $\bar{W}_{n}(\omega) \stackrel{\text { a.s. }}{\longrightarrow} A$.

In $\S 3$ we prove a more general strong law (Theorem 3.1) in which the condition (1.1) is replaced by the local version (3.1). The strong law rests on the existence of truncation constants $C_{n} \rightarrow \infty$ which satisfy the conditions (3.2) to (3.4). A canonical choice is $\widehat{C}_{n}=b_{n} / \omega_{n}$, where $\left(b_{n}\right)$ is a putative norming sequence. The condition $\sqrt{3.4}$, denoted by $\mathcal{K}(\mathbf{C})$, with $C_{n}=\widehat{C}_{n}$ (i.e., $\mathcal{K}(\widehat{\mathbf{C}})$ ) is our generalisation of (1.7) and it is dual to the weak law condition (2.5). The condition (3.3) is redundant in this case. Corollary 3.1 is an assertion about possible values of $\liminf _{n \rightarrow \infty} \bar{W}_{n}(\omega)$ if only $(3.2)$ and $(3.4)$ hold. This arises if it is desired to choose truncations such that $C_{n} / C_{n} \rightarrow 0$, a situation pertinent to Examples 2.1 and 2.2.

In $\$ 4$ we give some conditions which ensure (3.2) and (3.4). The essence of Lemma 4.2 is that if $C_{n} / \widehat{C}_{n} \rightarrow 0$, but not too quickly, then $\mathcal{K}(\widehat{\mathbf{C}})$ implies $\mathcal{K}(\mathbf{C})$. Theorem 4.1 gives conditions which entail more tractable versions of (3.2), and a partial converse implying lim $\sup _{n \rightarrow \infty} \bar{W}_{n}(\omega)=\infty$ almost surely.

Theorem 4.2 asserts that the weak law of Theorem 2.2 does not extend to a strong law. This applies to Case 1 of Examples 2.1 and 2.2. Cases 2 and 3 of these examples are re-examined as Examples 4.1 and 4.2. The weak law extends as a strong law in Case 2 except, perhaps, at the boundary separating Cases 2 and 3. Here the strong law may hold, or may not, depending on subsidiary conditions.

\section{THE WEAK LAW}

Our weak law is based on the following result which is a direct translation into our formulation of Khinchin's weak law for triangular arrays [6, Theorem 2, p. 140]. 
THEOREM K. The necessary and sufficient conditions to have $\bar{W}_{n}(\omega) \stackrel{p}{\rightarrow} 1$ and $\left\{\omega_{j} Y_{j} / b_{n}: 1 \leqslant j \leqslant n, n=1,2, \ldots\right\}$ comprising an infinitesimal array are that, for every $\xi>0$,

$$
\lim _{n \rightarrow \infty} \sum_{j=1}^{n} P\left(Y_{j}>\xi b_{n} / \omega_{j}\right)=0
$$

and

$$
\lim _{n \rightarrow \infty} b_{n}^{-1} \sum_{j=1}^{n} E\left(Y_{j} ; Y_{j} \leqslant \xi b_{n} / \omega_{j}\right)=1 .
$$

Denote the integrated tail functions of $F_{j}$ and $F$ by $I_{j}$ and $I$, respectively, e.g., $I(x)=\int_{0}^{x} \bar{F}(y) d y$. Thus the precise form of the condition 1.1 is that there is a natural number $j^{\prime}$ such that

$$
\lim _{x \rightarrow \infty} \sup _{j \geqslant j^{\prime}}\left|\frac{I_{j}(x)}{a_{j} I(x)}-1\right|=0 .
$$

If $\mu=\int_{0}^{\infty} \bar{F}(y) d y<\infty$, then this condition is equivalent to $E\left(Y_{j}\right)=\mu a_{j}$. Finally, recall that the relative stability $(1.3)$ is valid if and only if there is a slowly varying function $\ell(x)$ such that

$$
I(x) \sim \ell(x) \quad(x \rightarrow \infty) .
$$

Observe that $\ell$ is bounded away from zero and, without loss of generality, it can be assumed that $\ell$ is ultimately non-decreasing; we write $\ell \in S V_{\uparrow}$. With these preliminaries, our weak law is as follows.

THEOREM 2.1. Assume the conditions:

(i) 2.3) and (2.4) with $\ell \in S V_{\uparrow}$; and

(ii) $\lim _{n \rightarrow \infty} b_{n}^{-1} \max _{1 \leqslant j \leqslant n} \omega_{j}=0$.

Then $\bar{W}_{n}(\omega) \stackrel{p}{\rightarrow} 1$ if and only if

$$
\lim _{n \rightarrow \infty} b_{n}^{-1} \sum_{j=1}^{n} a_{j} \omega_{j} \ell\left(b_{n} / \omega_{j}\right)=1 .
$$

The following result is used in the proof of Theorem 2.1.

LEMMA 2.1. Suppose that the condition (ii) of Theorem 2.1 holds, that $\ell \in$ $S V$ and that $\xi>0$ is a constant. Then the condition

$$
\lim _{n \rightarrow \infty} b_{n}^{-1} \sum_{j=1}^{n} a_{j} \omega_{j} \ell\left(\xi b_{n} / \omega_{j}\right)=1
$$

is equivalent to (2.5). 
Proof. It suffices to prove that 2.5 implies 2.6. The condition (ii) implies that

$$
q_{n}:=\min _{1 \leqslant j \leqslant n}\left(b_{n} / \omega_{j}\right) \rightarrow \infty
$$

and hence, for any $\epsilon>0$, there exists a number $n_{\epsilon}>0$ such that $\ell\left(\xi b_{n} / \omega_{j}\right)$ is bounded between $(1 \pm \epsilon) \ell\left(b_{n} / \omega_{j}\right)$ if $1 \leqslant j \leqslant n$ and $n>n_{\epsilon}$. The assertion follows.

Proof of Theorem 2.1. The proof that 2.5 implies the weak law is in two parts.

(a) In our notation, the condition 2.1 is that $\lim _{n \rightarrow \infty} \sum_{j=1}^{n} \bar{F}\left(\xi b_{n} / \omega_{j}\right)=0$. Observe that

$$
I_{j}(x)-I_{j}(x / 2) \geqslant \frac{1}{2} x \bar{F}_{j}(x) .
$$

It follows from 2.3 that $I_{j}(x) \sim a_{j} I(x) \sim a_{j} \ell(x)$ and hence that $I_{j}(x)-$ $I_{j}(x / 2)=o\left(I_{j}(x)\right)$. We thus conclude, for each $j$, that $\bar{F}_{j}(x)=o\left(I_{j}(x) / x\right)$ as $x \rightarrow \infty$.

To see that this holds uniformly with respect to $j \geqslant j^{\prime}$ observe, for $\epsilon \in(0,1)$, that $(2.3)$ implies that there exists $x_{\epsilon}>0$ such that $I_{j}(x) \leqslant(1+\epsilon) a_{j} I(x)$ and $I_{j}(x / 2)>(1-\epsilon) a_{j} I(x / 2)$ if $x>x_{\epsilon}$ and $j \geqslant j^{\prime}$. Hence

$$
I_{j}(x)-I_{j}(x / 2) \leqslant a_{j}[(1+\epsilon) I(x)-(1-\epsilon) I(x / 2)] \sim 2 \epsilon a_{j} \ell(x) \quad(x \rightarrow \infty) .
$$

Thus $x_{\epsilon}$ can be chosen so large that

$$
\bar{F}_{j}(x) \leqslant 4(1+\epsilon) \epsilon a_{j} x^{-1} \ell(x) \quad\left(x>x_{\epsilon}, j \geqslant j^{\prime}\right) .
$$

It follows that if $n$ is such that $\xi q_{n}>x_{\epsilon}$, then

$$
\sum_{j^{\prime} \leqslant j \leqslant n} \bar{F}_{j}\left(\xi b_{n} / \omega_{j}\right) \leqslant 4(1+\epsilon)(\epsilon / \xi) b_{n}^{-1} \sum_{j^{\prime} \leqslant j \leqslant n} a_{j} \omega_{j} \ell\left(\xi b_{n} / \omega_{j}\right) .
$$

Clearly $\lim _{n \rightarrow \infty} \sum_{j=1}^{j^{\prime}} \bar{F}_{j}\left(\xi b_{n} / \omega_{j}\right)=0$, and hence we conclude, appealing to Lemma 2.1, that

$$
\limsup _{n \rightarrow \infty} \sum_{j=1}^{n} \bar{F}_{j}\left(\xi b_{n} / \omega_{j}\right) \leqslant 4(1+\epsilon)(\epsilon / \xi) .
$$

Letting $\epsilon \rightarrow 0$ thus yields 2.1 .

(b) Observe that $(2.2)$ is equivalent to

$$
\lim _{n \rightarrow \infty} b_{n}^{-1} \sum_{j=1}^{n} \omega_{j} E\left(Y_{j} ; Y_{j} \leqslant \xi b_{n} / \omega_{j}\right)=1 .
$$

Integration by parts yields the decomposition

$$
E\left(Y_{j} ; Y_{j} \leqslant x\right)=I_{j}(x)-x \bar{F}_{j}(x),
$$


implying a corresponding decomposition of the $n$-dependent terms in 2.7). The subtracted term contributes

$$
\xi \sum_{j=1} \bar{F}_{j}\left(\xi b_{n} / \omega_{j}\right) \rightarrow 0 \quad(n \rightarrow \infty)
$$

from part (a). Thus the leading contribution to the left-hand side of (2.7) equals $b_{n}^{-1} \sum_{j=1}^{n} I_{j}\left(\xi b_{n} / \omega_{j}\right)$. This quantity converges to unity because, as above, the sum over $j \in\left[j^{\prime}, n\right]$ is bounded between $(1 \pm \epsilon) b_{n}^{-1} \sum_{j=j^{\prime}}^{n} a_{j} \omega_{j} \ell\left(\xi b_{n} / \omega_{j}\right)$.

We conclude that 2.5 implies $\bar{W}_{n}(\omega) \stackrel{p}{\rightarrow} 1$. It is plain from the above estimates and Lemma 2.1 that the conditions (2.1) and 2.2) imply (2.5).

The uniform convergence theorem for slowly varying functions yields the following simplification of the condition (2.5) with the 'explicit' form (2.10) of the norming constants. It is a small generalisation of the Adler and Pakes [2] weak law. The (de Bruijn) conjugate function $\widehat{\ell}(x)$ of $1 / \ell(x)$ is the asymptotically unique slowly varying function satisfying (Bingham et al. [4, Theorem 1.5.13])

$$
\lim _{x \rightarrow \infty} \frac{\ell(x \widehat{\ell}(x))}{\widehat{\ell}(x)}=1, \quad \text { equivalently } \quad \lim _{x \rightarrow \infty} \frac{\widehat{\ell}(x / \ell(x))}{\ell(x)}=1 .
$$

COROLlaRY 2.1 .

(i) If $0<\inf _{j \geqslant 1} \omega_{j} \leqslant \sup _{j \geqslant 1} \omega_{j}<\infty$, then 2.5 is equivalent to the condition

$$
\lim _{n \rightarrow \infty} \mathcal{A}(n) \frac{\ell\left(b_{n}\right)}{b_{n}}=1, \quad \text { where } \mathcal{A}(n)=\sum_{j=1}^{n} a_{j} \omega_{j}
$$

In addition $\mathcal{A}(n) \rightarrow \infty$ and the norming sequence has the 'explicit' form

$$
b_{n}=\mathcal{A}(n) \widehat{\ell}(\mathcal{A}(n)) \text {. }
$$

(ii) If the weight sequence is arbitrary but $\mu:=\int_{0}^{\infty} \bar{F}(x) d x<\infty$, then 2.5 holds if $b_{n}=\mu \mathcal{A}(n)$.

This result suggests the plausible conjecture that the replacement of (2.5) with (2.9) will hold if the weighting sequence converges to zero (or infinity) 'sufficiently slowly'. Now what 'sufficiently slowly' really means will depend on the nature of $F$ and the sequence $\left(a_{j}\right)$. The following result pins this down in the still quite general case that there is a function $U(y)>0$ such that

$$
\ell(x)=U(\log x) \quad \text { and } \quad U(y+o(y)) \sim U(y) \quad(y \rightarrow \infty) .
$$

The second condition, written $U \in A N$, an asymptotic negligibility type of condition, is satisfied if, for example, $U$ is regularly varying. Subject to the restriction 
2.11, the next result can be regarded as a generalisation of Khinchin's weak law (reviewed by Adler and Pakes [1]). The proof is for the case $\omega_{n} \rightarrow 0$.

THEOREM 2.2 Suppose that $U \in A N$, that the weight sequence $\left(\omega_{j}\right)$ is ultimately monotone, that

$$
\mathcal{A}(n):=\sum_{j=1}^{n} a_{j} \omega_{j} \rightarrow \infty,
$$

and that the sequence $\left(b_{n}\right)$ is specified by 2.10. If

$$
\lim _{n \rightarrow \infty} \frac{\log \omega_{n}^{-1}}{\log \mathcal{A}(n)}=0,
$$

then the weak law holds with $b_{n} \sim \mathcal{A}(n) \ell(\mathcal{A}(n))$.

Proof. It follows from 2.10 and $\log \widehat{\ell}(x)=o(\widehat{\ell}(x))$ that $\log b_{n} \sim \log \mathcal{A}(n)$, and hence the hypotheses imply that

$$
\begin{aligned}
\ell\left(b_{n} / \omega_{j}\right) & =U\left(\log b_{n}+\log \omega_{j}^{-1}\right)=U\left(\log b_{n}(1+o(1)) \sim U(\log \mathcal{A}(n))\right. \\
& =\ell(\mathcal{A}(n)) .
\end{aligned}
$$

Consequently,

$$
\mathcal{C}(n):=\sum_{j=1}^{n} a_{j} \omega_{j} \ell\left(b_{n} / \omega_{j}\right)
$$

satisfies $\mathcal{C}(n) \sim \mathcal{A}(n) \ell(\mathcal{A}(n))$. Using the fact that $(\log \hat{\ell}(x)) / \log x \rightarrow 0$ (Bingham et al. [4, Proposition 1.3.6(i)]), we see from 2.11) that $\ell(x \widehat{\ell}(x))=$ $U((\log x)(1+o(1)) \sim \ell(x)$, i.e., the first member of 2.8$)$ is satisfied with $\widehat{\ell}(x)$ $\sim \ell(x)$. Hence 2.5 is satisfied and the assertion follows from Theorem 2.1.

Next we explore two examples where the limit (2.12) exists and is positive and the norming sequence does not have the form (2.10). In both examples we assume that $\ell$ has the form 2.11 in the more specific sense that

$$
\ell(x)=U_{\rho}(\log x)
$$

where $U_{\rho} \in R V_{\rho}$, the class of regularly varying functions with index $\rho \geqslant 0$. Denote the slowly varying factor of $U_{\rho}$ by $L_{U}(x)$, so $U_{\rho}(x)=x^{\rho} L_{U}(x)$. We use a similar notation for other regularly varying functions which may arise below. This emphasises our convention that the index of regular variation may change but the slowly varying factors do not.

Observe too that to assess 2.5 we need only the forms of the product $p_{n}=$ $a_{n} \omega_{n}$ and of $\log \omega_{n}^{-1}$. In the examples which follow we choose weights $\omega_{n} \rightarrow 0$. In addition the terms in sums such as $\mathcal{A}(n)$ comprise a regularly varying sequence 
and hence these sums are asymptotically equal to the analogous integral. These integrals are evaluated without comment using appropriate properties of regularly varying functions such as are set out in Bingham et al. [4, §1.6]. Our examples also involve iterates of the logarithm function. Following Adler and Pakes [2] (but altering their notation), we define functions $\log _{0}(x)=x, \log _{1}(x)=\log x$ and, for $\left.k=2,3, \ldots, \log _{k}(x)=\log \left(\log _{k-1}(x)\right)=\log _{k-1}(\log x)\right)$. These functions are defined for sufficiently large $x$ and this suffices since we are involved only with asymptotic estimates.

EXAMPLE 2.1. Let $A \in(0,1)$ and $\gamma \geqslant 0$ be constants and $R_{A-1} \in R V_{A-1}$. For $n \geqslant n^{\prime}>1$ we set

$$
p_{n}=n^{-1} R_{A-1}(\log n) \quad \text { and } \quad \log \omega_{n}^{-1} \sim Q_{\gamma}(\log n),
$$

where $Q_{\gamma} \in R V_{\gamma}$, and we assume that the weight sequence is ultimately decreasing to zero.

We have

$$
\begin{aligned}
\mathcal{A}(n) & \sim \sum_{j \leqslant n} j^{-1} R_{A-1}(\log j) \sim \int_{2}^{n} R_{A-1}(\log x) d \log x \\
& =\int_{\log 2}^{\log n} R_{A-1}(y) d y \sim A^{-1} R_{A}(\log n) .
\end{aligned}
$$

It follows that

$$
\log \mathcal{A}(n) \sim A \log _{2} n,
$$

CASE 1. We assume the condition (2.12) holds, in which case $\gamma=0$. It follows from Theorem 2.2 that the weak law holds with

$$
b_{n} \sim A^{-1} R_{A}(\log n) U_{\rho}\left(A \log _{2} n\right) \sim A^{\rho-1} R_{A}(\log n) U_{\rho}\left(\log _{2} n\right) .
$$

Now assume that $(2.12$ ) fails in the sense that

$$
C:=\lim _{n \rightarrow \infty} \frac{\log \mathcal{A}(n)}{\log \omega_{n}^{-1}} \in[0, \infty) .
$$

In what follows we use the notation $\mathcal{R}_{k, \theta}$ (with $k=1,2, \ldots$ ) to denote regularly varying functions of index $\theta$ which will be specified in terms of those introduced above. Only the index of regular variation will be significant.

Recalling from Remark 2.1 that

$$
\mathcal{B}(n)=\sum_{j=1}^{n} p_{j} \ell\left(\omega_{j}^{-1}\right),
$$


a computation similar to that for $\mathcal{A}(n)$ yields

$$
\begin{aligned}
\mathcal{B}(n) & \sim \int_{\log 2}^{\log n} R_{A-1}(y) U_{\rho}\left(Q_{\gamma}(y)\right) d y \\
& \sim(A+\rho \gamma)^{-1} R_{A}(\log n) U_{\rho}\left(Q_{\gamma}(\log n)\right)=: \mathcal{R}_{1, A+\rho \gamma}(\log n)
\end{aligned}
$$

We will see that 2.5 is satisfied in the case that the limit 2.12 is positive by taking $b_{n}$ proportional to $\mathcal{B}(n)$.

CASE 2: $C=0$. This case always holds if $\gamma>0$. In particular, a notion of asymptotically identical distribution is defined by choosing $a_{j}=1$ for all large $j$. In this case $\log \omega_{n}^{-1} \sim \log n$, i.e. $\gamma=1$. See Adler and Pakes [1] for the case where the $Y_{j}$ have the same law.

We will choose $b_{n} \sim \mathcal{B}(n)$ in which case

$$
\log b_{n} \sim(A+\rho \gamma) \log _{2} n
$$

Noting that the condition 2.16 is equivalent to $(\log x) / Q_{\gamma}(x) \rightarrow 0$, we will write $Q_{\gamma}(x)=(\log x) / h(x)$, where $h(x) \rightarrow 0$. Let $\left(j_{n}\right)$ denote a monotone sequence of positive integers satisfying $j_{n} \rightarrow \infty$ and $\log j_{n} \sim(\log n)^{\nu}$, where $\nu \in(0,1)$ is a constant. Clearly $j_{n} / n \rightarrow 0$. Also,

$$
\frac{\log b_{n}}{\log \omega_{j_{n}}^{-1}} \sim \frac{(A+\rho \gamma)}{Q_{\gamma}\left((\log n)^{\nu}\right)} \sim \nu^{-1}(A+\rho \gamma) h\left(j_{n}\right) \rightarrow 0
$$

Since $\log \omega_{j}^{-1}$ is ultimately non-decreasing, it follows that

$$
\frac{\log b_{n}}{\log \omega_{j}^{-1}} \rightarrow 0 \quad \text { if } j_{n}<j \leqslant n
$$

Hence

$$
\ell\left(b_{n} / \omega_{j}\right)=U_{\rho}\left(\log b_{n}+\log \omega_{j}^{-1}\right) \sim \ell\left(\omega_{j}^{-1}\right) \quad \text { if } j_{n}<j \leqslant n .
$$

Recalling the definition 2.13 , we see that

$$
\mathcal{C}(n)-\mathcal{C}\left(j_{n}\right)=\sum_{j_{n}<j \leqslant n} p_{j} \ell\left(b_{n} / \omega_{j}\right) \sim \sum_{j_{n}<j \leqslant n} p_{j} \ell\left(\omega_{j}^{-1}\right)=\mathcal{B}(n)-\mathcal{B}\left(j_{n}\right) .
$$

It follows from the above asymptotic form of $\mathcal{B}(n)$ that

$$
\mathcal{B}\left(j_{n}\right) \sim \mathcal{R}_{1, A+\rho \gamma}\left((\log n)^{\nu}\right) \sim \mathcal{R}_{2,(A+\rho \gamma) \nu}(\log n)=o(\mathcal{B}(n))
$$


Finally, it is clear for large $n$ that

$$
\begin{aligned}
\mathcal{C}\left(j_{n}\right) \leqslant \ell\left(b_{n} / \omega_{j_{n}}\right) \mathcal{A}\left(j_{n}\right) & \sim U_{\rho}\left(\log b_{n}+\log \omega_{j_{n}}^{-1}\right) A^{-1} R_{A}\left((\log n)^{\nu}\right) \\
& \sim U_{\rho}\left(Q_{\gamma}\left((\log n)^{\nu}\right) A^{-1} R_{A}\left((\log n)^{\nu}\right)\right. \\
& =\mathcal{R}_{3,(A+\rho \gamma) \nu}(\log n)=o(\mathcal{B}(n) .
\end{aligned}
$$

Hence 2.5 is satisfied by taking $b_{n} \sim \mathcal{B}(n)$, as asserted above.

REMARK 2.2. If $U_{\rho}(x) \sim x^{\rho}, R_{A}(x) \sim x^{A}$ and $Q_{\gamma}(x) \sim q x^{\gamma}$ where $q>0$, then

$$
b_{n} \sim(A+\rho \gamma)^{-1} q^{\rho \gamma}(\log n)^{A+\rho \gamma} .
$$

Corollary 1.1(i) in Nakata [8] is the case $A=b-1$ and $\rho=\gamma=1$.

CASE 3: $C>0$. This assumption requires that $\gamma=0$ and $Q_{0}(x) \sim(A / C) \log x$. It follows that $\log \omega_{j}^{-1} \sim(A / C) \log _{2} j$.

We will set $b_{n}=K \mathcal{B}(n)$ where $K$ is a constant to be determined. Choosing $\delta \in(0,1)$ and $j_{n} \sim n^{\delta}$, we observe that $\left(\log _{2}\left(n^{\delta}\right)\right) / \log _{2} n \rightarrow 1$ and, as above, $\log b_{n} \sim A \log _{2} n$. It follows from these observations that

$$
\frac{\log b_{n}}{\log \omega_{j}^{-1}} \rightarrow C \quad \text { if } n^{\delta}<j \leqslant n .
$$

Consequently,

$$
\ell\left(b_{n} / \omega_{j}\right)=U_{\rho}\left(\log b_{n}+\log \omega_{j}^{-1}\right) \sim(1+C)^{\rho} \ell\left(\omega_{j}^{-1}\right)
$$

and hence

$$
\mathcal{C}(n)-\mathcal{C}\left(j_{n}\right) \sim(1+C)^{\rho}\left(\mathcal{B}(n)-\mathcal{B}\left(j_{n}\right)\right) .
$$

But

$$
\mathcal{B}\left(j_{n}\right) \sim \mathcal{R}_{1, A}\left(\log n^{\delta}\right) \sim \delta^{A} \mathcal{R}_{1, A}(\log n) \sim \delta^{A} \mathcal{B}(n),
$$

implying that

$$
\mathcal{C}(n)-\mathcal{C}\left(j_{n}\right) \sim(1+C)^{\rho}\left(1-\delta^{A}\right) \mathcal{B}(n) .
$$

As above,

$$
\begin{aligned}
\mathcal{C}\left(j_{n}\right) \leqslant U_{\rho}\left(\log b_{n}+\log \omega_{n}^{-1}\right) \mathcal{A}\left(n^{\delta}\right) & \sim(1+C)^{\rho} U_{\rho}\left(Q_{0}(\log n)\right) \delta^{A} \mathcal{A}(n) \\
& =\delta^{A}(1+C)^{\rho} \mathcal{R}_{1, A}(\log n) \\
& \sim \delta^{A}(1+C)^{\rho} \mathcal{B}(n) .
\end{aligned}
$$

It follows that

$$
\begin{aligned}
K^{-1}(1+C)^{\rho}\left(1-\delta^{A}\right) & \leqslant \liminf _{n \rightarrow \infty} b_{n}^{-1} \mathcal{C}(n) \leqslant \limsup _{n \rightarrow \infty} b_{n}^{-1} \mathcal{C}(n) \\
& \leqslant K^{-1} \delta^{A}+K^{-1}(1+C)^{\rho}\left(1-\delta^{A}\right) .
\end{aligned}
$$


Let $\delta \rightarrow 0$ and choose $K=(1+C)^{\rho}$ to see that $\bar{W}_{n}(\omega) \stackrel{p}{\rightarrow} 1$ with

$$
b_{n} \sim A^{\rho-1}\left(1+C^{-1}\right)^{\rho} R_{A}(\log n) U_{\rho}\left(\log _{2} n\right) .
$$

REMARK 2.3. Taking account of the specific form of $Q_{0}$, the other parameters in Remark 2.2 yield $b_{n} \sim A^{\rho-1}\left(1+C^{-1}\right)^{\rho}(\log n)^{A}\left(\log _{2} n\right)^{\rho}$. Corollary 1.1(ii) in Nakata [8] is the case where $\rho=1, A=b$, and $Q_{0}(x)=(1-A) \log x$. These yield $C=b /(1-b)$, so $b_{n} \sim b^{-1}(\log n)^{b} \log _{2} n$.

The following result is a companion to Theorem 2.2. It gives different conditions under which 2.10) implies 2.5.

THEOREM 2.3. Suppose that $\left(b_{n}\right)$ is a sequence such that $b_{n} \rightarrow \infty$.

(i) If the weight sequence $\left(\omega_{j}\right)$ is monotone and

$$
\lim _{n \rightarrow \infty} \frac{\ell\left(b_{n} / \omega_{n}\right)}{\ell\left(b_{n}\right)}=1
$$

then 2.5 is equivalent to 2.9.

(ii) The condition 2.22 holds if $\omega_{n} \rightarrow 0\left(\right.$ or $\left.\omega_{n} \rightarrow \infty\right)$ and, in addition, the index function $\varepsilon_{\ell}(x)$ of $\ell$ is slowly varying and

$$
\lim _{n \rightarrow \infty} \varepsilon_{\ell}\left(b_{n}\right) \log \omega_{n}=0 .
$$

(iii) Suppose that $F$ is continuous, that $\ell \in S V_{N}$ and the index function $\varepsilon_{\ell}$ is ultimately monotone. Then the conditions (2.5) and (2.9) are equivalent if

$$
\lim _{n \rightarrow \infty} \bar{F}\left(b_{n}\right) \mathcal{B}_{n}=0 \text {. }
$$

If either of the conditions in (ii) or (iii) hold then the weak law holds with $b_{n}$ as in 2.10.

Proof. It suffices to assume that $\left(\omega_{n}\right)$ is decreasing. Since $\ell$ is non-decreasing, we have the inequality

$$
\ell\left(b_{n} / \omega_{1}\right) \leqslant \ell\left(b_{n} / \omega_{j}\right) \leqslant \ell\left(b_{n} / \omega_{n}\right) \quad(1 \leqslant j \leqslant n) .
$$

Assertion (i) follows from the slow variation of $\ell$.

For (ii), it follows from the integral representation of slowly varying functions that

$$
\log \frac{\ell\left(b_{n} / \omega_{n}\right)}{\ell\left(b_{n}\right)} \sim \int_{b_{n}}^{b_{n} / \omega_{n}}\left(\varepsilon_{\ell}(x) / x\right) d x=\int_{1}^{\omega_{n}^{-1}}\left(\varepsilon_{\ell}\left(b_{n} y\right) / y\right) d y=O\left(\varepsilon_{\ell}\left(b_{n}\right)\left|\log \omega_{n}\right| .\right.
$$

The assertion follows. 
(iii) If $\omega \in(0,1]$, then

$$
\begin{aligned}
\omega\left|\frac{\ell(x / \omega)}{\ell(x)}-1\right| & =\omega O\left(\exp \int_{x}^{x / \omega} \frac{\varepsilon_{\ell}(y)}{y} d y-1\right)=O\left(\omega \int_{x}^{x / \omega} \frac{\varepsilon_{\ell}(y)}{y} d y\right) \\
& =O\left(\varepsilon_{\ell}(x) \omega \log \omega^{-1}\right) .
\end{aligned}
$$

Since $\omega_{n} \downarrow 0$ we can assume that $\omega_{n} \leqslant 1$. So it follows from the above estimate that for any sequence $b_{n} \uparrow \infty$,

$$
\begin{aligned}
b_{n}^{-1}\left|\sum_{j=1}^{n} a_{j} \omega_{j} \ell\left(b_{n} / \omega_{j}\right)-\ell\left(b_{n}\right) \mathcal{A}(n)\right| & \leqslant \frac{\ell\left(b_{n}\right)}{b_{n}} \sum_{j=1}^{n} a_{j} \omega_{j}\left|\frac{\ell\left(b_{n} / \omega_{j}\right)}{\ell\left(b_{n}\right)}-1\right| \\
& \leqslant O\left(\frac{\ell\left(b_{n}\right)}{b_{n}} \varepsilon_{\ell}\left(b_{n}\right) \mathcal{B}_{n}\right) .
\end{aligned}
$$

The continuity assumption implies that $\ell^{\prime}(x)=\bar{F}(x)=\varepsilon_{\ell}(x)(\ell(x) / x)$. The assertion (iii) now follows.

The final assertion follows because $b_{n}$ given by (2.10) satisfies 2.9].

What is the relation between the conditions (2.12), 2.23) and 2.24)? Suppose that 2.10) does hold, in which case

$$
b_{n} / \ell\left(b_{n}\right) \sim \mathcal{A}(n) \text { and } \mathcal{B}(n) \sim \mathcal{A}(n) \ell\left(b_{n}\right)
$$

Observe that this norming depends on the weights only through $\mathcal{A}(n)$. For the simple case where, if $x$ is large and $\rho>0$, then $\ell(x)=(\log x)^{\rho}$, it follows that

$$
\varepsilon_{\ell}\left(b_{n}\right) \sim \rho / \log b_{n} \sim \rho / \log \mathcal{A}(n) .
$$

Hence (2.23) and 2.12) are equivalent conditions, and this can be expected to hold for more general forms of $\ell$, e.g., $\ell(x)=U_{\rho}(\log x)$ and the slowly varying factor is normalised slowly varying.

On the other hand, $\bar{F}(x)=\rho \ell(x) /(x \log x)$, hence $\bar{F}\left(b_{n}\right) \sim \rho /\left(\mathcal{A}(n) \log b_{n}\right)$ and

$$
\bar{F}\left(b_{n}\right) \mathcal{B}(n) \sim \rho\left(\log b_{n}\right)^{\rho-1} .
$$

The right-hand side tends to zero only if $\rho<1$. Thus the condition 2.24 has a quite limited range of applicability. In addition, it does not directly involve the weight sequence.

EXAMPLE 2.2. A slower rate of decrease of the $a_{j}$ 's and weights are obtained by altering Example 2.1 so that

$$
p_{n}=n^{\alpha-1} R_{A}(\log n),
$$

where $A$ is real and $\alpha \in(0,1)$. 
We now have

$$
\mathcal{A}(n) \sim \alpha^{-1} n^{\alpha} R_{A}(\log n) \text { and } \log \mathcal{A}(n) \sim \alpha \log n .
$$

CASE 1: If $\gamma \leqslant 1$ and $Q_{\gamma}(x) / x \rightarrow 0$, then Theorem 2.2 is applicable with

$$
b_{n} \sim \alpha^{\rho-1} n^{\alpha} R_{A}(\log n) U_{\rho}(\log n) .
$$

CASE 2: If $\gamma \geqslant 1$ and $Q_{\gamma}(x) / x \rightarrow \infty$, then the weak law holds with

$$
b_{n} \sim \mathcal{B}(n) \sim \alpha^{-1} n^{\alpha} R_{A}(\log n) U_{\rho}\left(Q_{\gamma}(\log n)\right) .
$$

The proof is similar to that for Case 2 in Example 2.1 using the auxiliary sequence $j_{n} \sim n^{\delta}$ where $\delta \in(0,1)$.

CASE 3: Here $\gamma=1$ so $Q_{1}(x) \sim(\alpha / C) x$ and 2.16) holds with $C \in(0, \infty)$. Using the auxiliary sequence $j_{n} \sim \delta n$ with $\delta \in(0,1)$, a similar argument to that used above for Case 3 of Example 2.1 yields the weak law with

$$
b_{n} \sim \alpha^{\rho-1}\left(1+C^{-1}\right)^{\rho} n^{\alpha} R_{A}(\log n) U_{\rho}(\log n) .
$$

Case 3 with $C=\alpha /(1-\alpha)$ occurs for asymptotically identical distributions.

\section{THE STRONG LAW}

The following strong law reflects Theorem 4.1 in Adler and Pakes [2] who give conditions in the case that $\omega_{n} \equiv 1$ which ensure that $\liminf _{n \rightarrow \infty} \bar{W}_{n}=1$ almost surely. We need to assume a more stringent version of (2.3), i.e., there exists a positive integer $j^{\prime}$ such that

$$
\lim _{x \rightarrow \infty} \sup _{j \geqslant j^{\prime}}\left|\frac{\bar{F}_{j}(x)}{a_{j} \bar{F}(x)}-1\right|=0 .
$$

THEOREM 3.1. Suppose that the $Y_{j}$ are independent with distribution functions $F_{j}$ satisfying 3.1 with $F \in R S$ and let $\widehat{C}_{j}=b_{j} / \omega_{j}$. Suppose in addition that there are sequences $\left(b_{n}\right)$ and $\mathbf{C}=\left(C_{n}\right)$ of positive numbers, both diverging to infinity, such that the following three conditions hold:

$$
\begin{aligned}
& S(\mathbf{C}):=\sum_{j=1}^{\infty} \widehat{C}_{j}^{-2} a_{j} \int_{0}^{C_{j}} x \bar{F}(x) d x<\infty ; \\
& \inf _{n \geqslant n^{\prime}} C_{n} / \widehat{C}_{n}>0 ; \\
& \lim _{n \rightarrow \infty} b_{n}^{-1} \sum_{j=1}^{n} a_{j} \omega_{j} \ell\left(C_{j}\right)=1 .
\end{aligned}
$$

Then $\bar{W}_{n}(\omega) \stackrel{\text { a.s. }}{\longrightarrow} 1$. 
Proof. We truncate $Y_{j}$ at level $C_{j}$ and resolve our average into three components,

$$
\bar{W}_{n}(\omega)=K_{1}(n)+K_{2}(n)+K_{3}(n),
$$

where the right-hand side summands will be defined as we go.

First, it follows from the Kolmogorov-Khinchin convergence theorem (Chow and Teicher [5, p. 110]) and Kronecker's lemma that

$$
K_{1}(n):=b_{n}^{-1} \sum_{j=1}^{n} \omega_{j}\left[Y_{j} 1\left(Y_{j} \leqslant C_{j}\right)-E\left(Y_{j} 1\left(Y_{j} \leqslant C_{j}\right)\right)\right] \stackrel{\text { a.s. }}{\longrightarrow} 0
$$

if the series

$$
S:=\sum_{j=1}^{\infty} \widehat{C}_{j}^{-2} E\left[Y_{j}^{2} 1\left(Y_{j} \leqslant C_{j}\right)\right]
$$

converges. The expectation equals

$$
\int_{0}^{C_{j}} x^{2} d F_{j}(x)=2 \int_{0}^{C_{j}} x \bar{F}_{j}(x) d x-C_{j}^{2} \bar{F}_{j}\left(C_{j}\right)=O\left(a_{j} \int_{0}^{C_{j}} x \bar{F}(x) d x\right),
$$

where the final estimate comes by virtue of (3.1). Hence (3.2) implies that $S<\infty$, so $K_{1}(n) \stackrel{\text { a.s. }}{\longrightarrow} 0$.

Second, the previous estimate implies that

$$
C_{j}^{2} P\left(Y_{j} \geqslant C_{j}\right) \leqslant 2 \int_{0}^{C_{j}} x \bar{F}_{j}(x) d x
$$

so it follows from $\sqrt{3.3}$ and $S<\infty$ that $\sum_{j \geqslant 1} P\left(Y_{j}>C_{j}\right)<\infty$. Hence the Borel-Cantelli lemma implies there is a random variable $\mathcal{N}$ such that almost surely $Y_{j} \leqslant C_{j}$ if $j \geqslant \mathcal{N}$. Hence

$$
K_{2}(n):=b_{n}^{-1} \sum_{j=1}^{n} \omega_{j} Y_{j} 1\left(Y_{j}>C_{j}\right) \leqslant b_{n}^{-1} \sum_{j=1}^{\mathcal{N}} \omega_{j} C_{j} \stackrel{\text { a.s. }}{\longrightarrow} 0 .
$$

Finally,

$$
\begin{aligned}
K_{3}(n) & :=b_{n}^{-1} \sum_{j=1}^{n} \omega_{j} E\left[Y_{j} 1\left(Y_{j} \leqslant C_{j}\right)\right] \\
& =b_{n}^{-1} \sum_{j=1}^{n} \omega_{j} \int_{0}^{C_{j}} x d F_{j}(x)=K_{13}(n)-K_{23}(n),
\end{aligned}
$$

where

$$
K_{13}(n)=b_{n}^{-1} \sum_{j=1}^{n} \omega_{j} \int_{0}^{C_{j}} \bar{F}_{j}(x) d x .
$$

The assumption (3.1) implies 2.3 and hence the integral is asymptotically equal to $a_{j} \ell\left(C_{j}\right)$. Consequently, 3.4 implies that $\lim _{n \rightarrow \infty} K_{13}(n)=1$. 
The remaining term is

$$
K_{23}(n):=b_{n}^{-1} \sum_{j=1}^{n} \omega_{j} C_{j} \bar{F}_{j}\left(C_{j}\right) \sim b_{n}^{-1} \sum_{j=1}^{n} a_{j} \omega_{j} C_{j} \bar{F}\left(C_{j}\right)=o\left(K_{13}(n)\right),
$$

where the last equality follows because $F \in R S$ is equivalent to the condition $x \bar{F}(x)=o(\ell(x))$; see Bingham et al. [4, Theorem 8.8.1(i, ii, viii)]. Hence $K_{3}(n)$ $\rightarrow 1$, and the assertion follows.

If the strong law is valid, then so is the weak law and hence 2.5 must hold. So this condition will, as seen in the previous section, give the correct form of norming constants which can be used to seek truncation constants which will satisfy the conditions (3.2)-(3.4). The condition (3.3) obviously holds for the canonical truncation $C_{j}=C_{j}$. The convergence condition (3.1) simplifies with more stringent assumptions about $\bar{F}$; see Theorem 4.1 .

Theorem 4.1 in Adler and Pakes [2] specifies numbers $d_{j}$ such that $C_{j}=a_{j} d_{j}$ and their conditions (4.2) and (4.1) correspond, respectively, to 3.2 and 3.4 above in the case that $\omega_{j} \equiv 1$. Finally, we note that, as shown by Adler and Pakes [2], there may not be an almost sure strengthening of the weak law.

The following corollary extends Theorem 4.1 of Adler and Pakes [2]. Its proof strategy is the same as that in this reference, i.e., observing that $\bar{W}_{n}(\omega) \geqslant K_{1}(n)+$ $K_{3}(n)$. The outcome (ii) follows from (i) and the weak law because the latter implies that $\liminf \operatorname{in}_{n \rightarrow \infty} \bar{W}_{n}(\omega) \leqslant 1$ almost surely.

COROllary 3.1. Suppose that (3.1) holds with $F \in R S$. Suppose in addition that there are sequences $\left(b_{n}\right)$ and $\left(C_{n}\right)$ of positive numbers, both diverging to infinity, such that (3.2) and 3.4 both hold. Then

$$
\liminf _{n \rightarrow \infty} \bar{W}_{n}(\omega) \geqslant 1 \quad \text { almost surely }
$$

and if also $\bar{W}_{n}(\omega) \stackrel{p}{\rightarrow} 1$, then

$$
\liminf _{n \rightarrow \infty} \bar{W}_{n}(\omega)=1 \quad \text { almost surely. }
$$

In the next section we will look at consequences of Theorem 3.1.

\section{CASES OF THEOREM 3.1}

Denote the condition 3.4 by $\mathcal{K}(\mathbf{C})$. A plausible conjecture is that the condition 2.5 and $\mathcal{K}(\widehat{\mathbf{C}})$ are equivalent, perhaps with the addition of supplementary assumptions. It is certainly obvious that if $(2.5)$ holds, then the lim sup of the average at 3.4 with $C_{j}=\widehat{C}_{j}$ is bounded above by unity. The following result, related to Theorem 4.1(ii) of Adler and Pakes [2], asserts that (2.5) implies (3.4) if (2.12) holds. 
LEMMA 4.1. Suppose that the assumptions of Theorem 2.2 are satisfied and that $\widehat{C}_{j} \rightarrow \infty$. Then the condition $\mathcal{K}(\widehat{\mathbf{C}})$ holds.

Proof. Exactly as in the proof of Theorem 2.2,

$$
\ell\left(b_{j} / \omega_{j}\right) \sim \ell\left(b_{j}\right) \sim \ell(\mathcal{A}(j))
$$

implying that

$$
\sum_{j=1}^{n} a_{j} \omega_{j} \ell\left(b_{j} / \omega_{j}\right) \sim \sum_{j=1}^{n} a_{j} \omega_{j} \ell\left(b_{j}\right) \sim \sum_{j=1}^{n}[\mathcal{A}(j)-\mathcal{A}(j-1)] \ell(\mathcal{A}(j)),
$$

where we define $\mathcal{A}(0)=0$. Extending the definition of $\mathcal{A}(\cdot)$, by linear interpolation say, to a monotone continuous function on $[0, \infty)$, we see that the last sum is an approximating sum of, and asymptotically equal to, the Stieltjes integral

$$
\int_{1}^{n} \ell(\mathcal{A}(z)) d \mathcal{A}(z)=\int_{\mathcal{A}(1)}^{\mathcal{A}(n)} \ell(x) d x \sim \mathcal{A}(n) \ell(\mathcal{A}(n)) \sim b_{n}
$$

recalling that $\widehat{\ell}(x) \sim \ell(x)$.

The next result emphasises that truncation sequences satisfying $\mathcal{K}(\mathbf{C})$ in general are not asymptotically unique. We omit the simple proof.

LEMmA 4.2. Suppose that $\widehat{C}_{j} \rightarrow \infty$ and that $\ell(x)=U(\log x)$ where $U \in$ $A N$. Let $C_{j}=\widehat{C}_{j} / M_{j}$ where $\left(M_{j}\right)$ is a positive sequence such that $C_{j} \rightarrow \infty$ and $\log M_{j}=o\left(\log \widehat{C}_{j}\right)$. Then $\mathcal{K}(\widehat{\mathbf{C}})$ implies $\mathcal{K}(\mathbf{C})$.

Next we consider the series $S(\mathbf{C})$. Observe that if $\mathbf{C}_{1} \leqslant \mathbf{C}_{2}$, where the inequalities are interpreted termwise, then $S\left(\mathbf{C}_{1}\right) \leqslant S\left(\mathbf{C}_{2}\right)$. The following result gives some simpler expressions of $S(\mathbf{C})<\infty$ under additional conditions and also a converse assertion in the event that $S(\mathbf{C})=\infty$. This assertion (in part (iii)) echos Theorem 3.1 in Adler and Pakes [2].

THEOREM 4.1 Suppose that 3.1 holds and that

$$
\bar{F}(x)=x^{-1} L(x),
$$

where $L \in S V$.

(i) If $C_{j} \rightarrow \infty$, then 3.2 is equivalent to

$$
S^{\prime}(\mathbf{C}):=\sum_{j=1}^{\infty} \widehat{C}_{j}^{-2} a_{j} C_{j} L\left(C_{j}\right)<\infty .
$$


(ii) If, in addition,

$$
\sup _{n \geqslant n^{\prime}} C_{n} / \widehat{C}_{n}<\infty
$$

then $S^{\prime}(\mathbf{C})<\infty$ if

$$
S^{\prime \prime}(\mathbf{C}):=\sum_{j=1}^{\infty}\left(a_{j} / C_{j}\right) L\left(C_{j}\right)<\infty .
$$

In particular, $S(\widehat{\mathbf{C}})<\infty$ if and only if $S^{\prime \prime}(\widehat{\mathbf{C}})<\infty$.

(iii) Conversely, if (4.1) and (3.3) both hold and if $S^{\prime \prime}(\mathbf{C})=\infty$, then

$$
\limsup _{n \rightarrow \infty} \omega_{n} Y_{n} / b_{n}=\infty \quad \text { almost surely. }
$$

In particular, $\lim \sup _{n \rightarrow \infty} \bar{W}_{n}(\omega)=\infty$.

(iv) If 4.1 holds and $S^{\prime \prime}(\widehat{\mathbf{C}})=\infty$, then $\limsup _{n \rightarrow \infty} \bar{W}_{n}(\omega)=\infty$ almost surely.

Proof. The slow variation of $L$ implies that the integral in $(3.2)$ is asymptotically equal to $C_{j} L\left(C_{j}\right)$ and the first assertion follows. The condition 4.4 is an obvious consequence of (4.2) and 4.3).

To prove the converse, if $S^{\prime \prime}(\widehat{\mathbf{C}})=\infty$ then, since $L$ is slowly varying, it follows that for any positive constant $M$ we have $\sum\left(a_{j} / C_{j}\right) L\left(M C_{j}\right)=\infty$. Hence 4.1) implies that $\sum_{j} P\left(Y_{j}>M C_{j}\right)=\infty$ so from the second Borel-Cantelli lemma, $P\left(Y_{n}>M C_{n}\right.$ i.o. $)=1$. Since $M$ is arbitrary, it follows from 3.3 that $\lim \sup \omega_{n} Y_{n} / b_{n}=\infty$, and (iii) follows. Assertion (iv) follows from (iii) because 3.3 holds if $C_{j}=\widehat{C}_{j}$.

REMARK 4.1. If $\bar{F}(x) \sim$ const/ $x$, in essence the assumption in Nakata [8], then the condition $S^{\prime \prime}(\widehat{\mathbf{C}})<\infty$ is the same as his condition $(1.6)$ above.

In all that follows we will assume that $F$ has the form 4.1 and that

$$
L(x) \sim \operatorname{const} \cdot \frac{U(\log x)}{\log x} \text { and } U \in A N .
$$

The monotone density theorem (Bingham et al. [4, p. 39]) implies this condition if $U(y) \in R V_{\rho}$ has an ultimately monotone derivative. In this case the constant in 4.5 equals $\rho$. We assume also that $\widehat{C}_{j} \rightarrow \infty$ and that $C_{j}=\widehat{C}_{j} / M(j)$ where $M(x)$ will be a positive-valued function which satisfies $\log M(j)=o\left(\log \widehat{C}_{j}\right)$.

These conditions together with Theorem 4.1 imply that

$$
L\left(C_{j}\right) \sim \frac{U\left(\log \widehat{C}_{j}\right)}{\log \widehat{C}_{j}}
$$


and hence that

$$
S(\mathbf{C})<\infty \quad \text { if and only if } \quad \widetilde{S}(\mathbf{M}):=\sum_{j=1}^{\infty} \frac{p_{j}}{b_{j}} \cdot \frac{U\left(\log \widehat{C}_{j}\right)}{M(j) \log \widehat{C}_{j}}<\infty .
$$

The following result shows that, assuming (4.1) and (4.5), the weak law of Theorem 2.2 does not extend to a strong law. Instead, we have the two-sided behaviour reported by Adler and Pakes [2] in the unweighted case.

THEOREM 4.2. Suppose that (4.1), 4.5) and the assumptions of Theorem 2.2 hold. Then, almost surely,

$$
\liminf _{n \rightarrow \infty} \bar{W}_{n}(\omega)=1 \quad \text { and } \quad \limsup _{n \rightarrow \infty} \bar{W}_{n}(\omega)=\infty .
$$

Proof. Recalling that the norming sequence for the weak law is (2.10), and that $\log b_{n} \sim \log \mathcal{A}(n)$, it follows from 2.12) that $U\left(\log \widehat{C}_{j}\right) \sim \ell(\mathcal{A}(j))$. Recalling next that $\ell(x) \sim \widehat{\ell}(x)$, it follows that the terms $\sigma_{j}(\mathbf{M})$ of the series $\widetilde{S}(\mathbf{M})$ satisfy

$$
\sigma_{j}(\mathbf{M}) \sim \frac{p_{j}}{\mathcal{A}(j) \widehat{\ell}(\mathcal{A}(j))} \cdot \frac{\ell(\mathcal{A}(j))}{M(j) \log \mathcal{A}(j)} \sim \frac{p_{j}}{M(j) \mathcal{A}(j) \log \mathcal{A}(j)} .
$$

Setting $M(x) \equiv 1$, we see from an integral test that $\widetilde{S}(\mathbf{M})<\infty$ if and only if

$$
\int_{1}^{\infty} \frac{d \mathcal{A}(x)}{\mathcal{A}(x) \log \mathcal{A}(x)}<\infty
$$

But it is obvious that this integral diverges, hence $S(\widehat{\mathbf{C}})=\infty$ and the lim sup assertion follows.

Now choose $\delta>0$ and $M(j) \sim(\log \mathcal{A}(j))^{\delta}$. Then $\log M(j) \sim \delta \log _{2} \mathcal{A}(j)=$ $o\left(\log \widehat{C}_{j}\right)$. In addition, the integral test implies that $\widetilde{S}(\mathbf{M})<\infty$ and hence the lim inf assertion follows from Corollary 3.1(ii).

In essence, this theorem implies that there is no strong law corresponding to the weak law if the weights converge too slowly to zero, in the sense of (2.12) for example. Theorem 4.2 obviously encompasses Case 1 of Examples 2.1 and 2.2.

We now re-examine Cases 2 and 3 of these examples and find that the strong law holds, aside from boundary cases. We do this by verifying (3.2) and 3.4) for the truncation sequence $\widehat{\mathbf{C}}$ where the norming sequence is that which delivers the weak law in these examples. It will turn out that the strong law can fail for a critical value of the parameter $\gamma$, but that two-sided behaviour similar to that in Theorem 4.2 can be established.

In all that follows we assume (4.1) and (4.5) with $U=U_{\rho}$ possessing an ultimately monotone derivative, implying $L(x) \sim U_{\rho}^{\prime}(\log x) \sim \rho U_{\rho}(\log x) / \log x$. 
EXAMPLE 4.1. Recall the specifications of Example 2.1 and assume that 2.16 holds. To see that 2.5 implies the condition $\mathcal{K}(\widehat{\mathbf{C}})$, argue as follows. Recalling the auxiliary sequence $j_{n}$ specified for Case 2, it follows from 2.20) that

$$
\log b_{j} / \log \omega_{j_{n}} \rightarrow 0 \quad \text { if } j_{n}<j \leqslant n \text { and } n \rightarrow \infty,
$$

and hence that $\ell\left(b_{j} / \omega_{j}\right) / \ell\left(\omega_{j}^{-1}\right) \rightarrow 1$ if $j_{n}<j \leqslant n$. This suffices to conclude that 2.5 implies $\mathcal{K}(\widehat{\mathbf{C}})$.

Recall for Case 3 that $\gamma=0$ and observe that 2.21) implies $\log b_{n} \sim A \log _{2} n$. Hence, choosing $j_{n} \sim n^{\delta}$, we find that $\log b_{j_{n}} \sim \log b_{n}$. Since $\log \omega_{j}^{-1} \sim$ $(A / C) \log _{2} j$, it follows that $\log b_{j} / \log \omega_{j} \rightarrow C$ if $n^{\delta}<j \leqslant n$, and hence $\mathcal{K}(\widehat{\mathbf{C}})$ holds.

In both cases, $b_{j} \sim$ const $\cdot R_{A}(\log j) U_{\rho}(Q(\gamma(\log j))$ and hence some algebraic reduction leads to the estimate

$$
\sigma_{j}(\mathbf{M}) \sim \frac{\text { const }}{j \log j Q_{\gamma}(\log j) M(j)} .
$$

We conclude for this example that the strong law holds if $\gamma>0$, or if $\gamma=0$ and

$$
I_{Q}:=\int_{1}^{\infty} \frac{d y}{y L_{Q}(y)}<\infty .
$$

This condition may hold, or may not hold, depending on the exact asymptotic form of $L_{Q}$. In Case 3, we know that $L_{Q}(x) \propto \log x$, and hence (4.9) cannot hold. Thus a further conclusion is that: If $\gamma=0$ and the integral in 4.9 ) diverges, then $\limsup _{n \rightarrow \infty} \bar{W}_{n}(\omega)=\infty$ almost surely.

So, suppose that $\gamma=0$ and $I_{Q}=\infty$ and that

$$
\limsup _{y \rightarrow \infty} \frac{\log _{2} y}{L_{Q}(y)}=0
$$

a condition always holding for Case 3 . Let $\delta>1$ and $N(y)=(\log y)\left(\log _{2} y\right)^{\delta}$ for $y \geqslant e$, set $M(x)=N(\log x)$ and in Lemma 4.2 choose $M_{j}=M(j)$. Since $\log \widehat{C}_{j} \propto \log \omega_{j}^{-1} \sim L_{Q}(\log j)$, the negligibility assumption in Lemma 4.2 is equivalent to $\lim _{y \rightarrow \infty} \log N(y) / L_{Q}(y)=0$, and this is implied by 4.10). Hence $\mathcal{K}(\mathbf{C})$ holds.

The condition 4.10 implies that for any $\epsilon>0$, there exists $y_{\epsilon}>0$ such that $1 / L_{Q}(y) \leqslant \epsilon / \log _{2} y$. Hence the integral

$$
\begin{aligned}
J(N) & :=\int_{e}^{\infty} \frac{d x}{(x \log x) L_{Q}(\log x) N(\log x)}=\int_{1}^{\infty} \frac{d y}{y L_{Q}(y) N(y)} \\
& \leqslant O(1)+\int_{y_{\epsilon}}^{\infty} \frac{d y}{y(\log y)\left(\log _{2} y\right)^{\delta}}<\infty
\end{aligned}
$$


i.e., $S(\mathbf{C})<\infty$. So we conclude from Corollary 3.1 that: If $\gamma=0, I_{Q}=\infty$ and (4.10) holds, then 4.7) is valid. This holds for Case 3.

Suppose now that 4.10 is replaced with the (not quite converse) condition

$$
\liminf _{y \rightarrow \infty} \frac{\log _{2} y}{L_{Q}(y)} \geqslant B \in(0, \infty) .
$$

Writing $M(x)=N(\log x)$ for some positive-valued function $N$, the condition $J(N)<\infty$ and 4.11 imply that for any large number $y_{B}$,

$$
\int_{y_{B}}^{\infty} \frac{d y}{y\left(\log _{2} y\right) N(y)}<\infty
$$

This can hold only if $N(y) \geqslant$ const $\log y$. But this will contradict the negligibility condition required for Lemma 4.2. Hence the strongest conclusion we can draw for this case is that: If $\gamma=0, I_{Q}=\infty$ and (4.11) holds, then almost surely

$$
\liminf _{n \rightarrow \infty} \bar{W}_{n}(\omega) \leqslant 1 \quad \text { and } \quad \limsup _{n \rightarrow \infty} \bar{W}_{n}(\omega)=\infty .
$$

REMARK 4.2. The strong law holds for the specifications of Remark 2.2 and $\gamma>0$. This subsumes Corollary 1.2 of Nakata [8] where $\gamma=1$. If $\gamma=0$ then, since $L_{Q}(x) \equiv q$ the integral (at (4.9p) $I_{Q}=\infty$. In addition the condition 4.10) fails, but (4.11) holds. Hence our best conclusion is that (4.12) holds.

REMARK 4.3. The configuration of Remark 2.3 corresponds to a Case 3 instance of Example 2.1. The condition (4.10) fails but (4.11) is satisfied and hence we conclude that 4.12 holds. This outcome holds for Corollary 1.1(ii) in Nakata [8] for which case he could conclude only that the strong law fails. Instead, following the general prescription in Adler and Wittmann [3], he constructed a modified weight sequence which yields a strong law.

EXAMPLE 4.2. Recall the specifications for Example 2.2 and assume that $\gamma \geqslant$ 1 , a necessary condition for Cases 2 and 3. Arguing as for Example 4.1 it will follow that $\mathcal{K}(\widehat{\mathbf{C}})$ holds. With reference to 4.6 , it follows from 2.25 and 2.26 that

$$
\sigma_{j}(\mathbf{M}) \sim \frac{\text { const }}{j Q_{\gamma}(\log j) M(j)} .
$$

Hence $\widetilde{S}(\mathbf{1})<\infty$ if $\gamma>1$ or if $\gamma=1$ and

$$
I=\int_{1}^{\infty} \frac{d y}{Q_{1}(y)}<\infty
$$

Hence the strong law holds in these cases. 
In the case that $\gamma=1$ and $I=\infty$ we choose $\delta>0$ and $M(j) \sim(\log j)^{\delta}$. Since $\log \widehat{C}_{j} \propto \log \omega_{j}^{-1} \sim Q_{1}(\log j)$, it follows that $\log M(j) / \log \widehat{C}_{j} \rightarrow 0$ and hence, from Lemma 4.2 , the condition $\mathcal{K}(\mathbf{C})$ holds. In addition, $\widehat{S}(\mathbf{M})<\infty$. It follows that (4.7) holds.

Acknowledgments. I thank the referee for a diligent reading of the manuscript and a suggestion resulting in a more compelling proof of Theorem 2.1.

\section{REFERENCES}

[1] A. Adler and A. G. Pakes, On relative stability and weighted laws of large numbers, Extremes 20 (2017), 1-31.

[2] A. Adler and A. G. Pakes, Weak and one-sided strong laws for random variables with infinite mean, Statist. Probab. Lett. 142 (2018), 8-16.

[3] A. Adler and R. Wittmann, Stability of sums of independent random variables, Stoch. Processes Appl. 52 (1994), 179-182.

[4] N. H. Bingham, C. M. Goldie and J. L. Teugels, Regular Variation, Cambridge Univ. Press, Cambridge 1987.

[5] Y. S. Chow and H. Teicher, Probability Theory: Independence, Interchangeability, Martingales, Springer, New York 1978.

[6] B. V. Gnedenko and A.N. Kolmogorov, Limit Distributions for Sums of Independent Random Variables, Addison-Wesley, Reading, MA, 1954.

[7] T. Nakata, Weak laws of large numbers for weighted independent random variables with infinite mean, Statist. Probab. Lett. 109 (2016), 124-129.

[8] T. Nakata, Exact laws of large numbers for independent Pareto random variables, Bull. Inst. Math. 12 (2017), 325-338.

Anthony G. Pakes

Department of Mathematics \& Statistics

University of Western Australia

35 Stirling Highway, Crawley WA, Australia 6009

E-mail: tony.pakes@uwa.edu.au 
